\title{
CONFORMAL EQUIVALENCE OF COUNTABLE DENSE SETS
}

\author{
W. D. MAURER
}

In [1, p. 297, problem 24], Erdös asks:

"Does there exist an entire function $f$, not of the form $f(z)=a_{0}+a_{1} z$, such that the number $f(x)$ is rational or irrational according as $x$ is rational or irrational? More generally, if $A$ and $B$ are two denumerable, dense sets, does there exist an entire function which maps $A$ on to $B$ ?"

The following theorem settles the second part of this question as it is stated.

Theorem. Let $A$ and $B$ be two countable dense sets in the complex plane. Then there exists an entire function taking $A$ onto $B$.

Proof. Let $a$ and $b$ be enumerations of $A$ and $B$, i.e., $A$ $=\left\{a_{1}, a_{2}, \cdots\right\}, B=\left\{b_{1}, b_{2}, \cdots\right\}$. We construct two new enumerations $c$ and $d$ of $A$ and $B$ respectively, together with a sequence $f_{i}$ of polynomials such that $f\left(c_{i}\right)=d_{i}$ for each $i$, where $f=\sum_{j=1}^{\infty} f_{j}$.

The construction is as follows. Let $c_{1}=a_{1}, d_{1}=b_{1}, f_{1}=d_{1}$ (the constant function). At the $(2 n-1)$ st stage, suppose that $c_{1}, \cdots, c_{2 n-1}$; $d_{1}, \cdots, d_{2 n-1}$; and $f_{1}, \cdots, f_{2 n-1}$ have been chosen, such that $g_{2 n-1}\left(c_{i}\right)$ $=d_{i}, 1 \leqq i \leqq 2 n-1$, where $g_{2 n-1}=\sum_{j=1}^{2 n-1} f_{j}$. Let $c_{2 n}=a_{j}$, where $j$ is the smallest index such that $a_{j} \neq c_{i}, 1 \leqq i \leqq 2 n-1$, and set $y_{2 n}=g_{2 n-1}\left(c_{2 n}\right)$. Let the function $h_{2 n-1}=\left(z-c_{1}\right)\left(z-c_{2}\right) \cdots \cdots\left(z-c_{2 n-1}\right)$, and consider the functions $g_{2 n-1}+k_{2 n-1} h_{2 n-1}$, for

$$
\left|k_{2 n-1}\right|<\frac{1}{(2 n-1) ! u_{1} u_{2} \cdot \cdots \cdot u_{2 n-1}}=m_{2 n-1}
$$

where $u_{i}=\max \left(1,\left|c_{i}\right|\right), 1 \leqq i \leqq 2 n-1$. These functions map $c_{2 n}$ into $\left\{y_{2 n}+k_{2 n-1} h_{2 n-1}\left(c_{2 n}\right):\left|k_{2 n-1}\right|<m_{2 n-1}\right\}$, which is a neighborhood of $y_{2 n}$ because $h_{2 n-1}\left(c_{2 n}\right) \neq 0$, and consequently contains an element of the dense set $B-\left\{d_{1}, \cdots, d_{2 n-1}\right\}$, which we denote by $d_{2 n}$. For the corresponding value of $k_{2 n-1}$, we set $f_{2 n}=k_{2 n-1} h_{2 n-1}, g_{2 n}=g_{2 n-1}+f_{2 n}$, which clearly implies $g_{2 n}\left(c_{i}\right)=d_{i}, 1 \leqq i \leqq 2 n$, and $g_{2 n}=\sum_{j=1}^{2 n} f_{j}$. This brings us to the $2 n$th stage. Let $d_{2 n+1}=b_{j}$, where $j$ is the smallest index such that $b_{j} \neq d_{i}, 1 \leqq i \leqq 2 n$, and set $x_{2 n+1}$ such that $g_{2 n}\left(x_{2 n+1}\right)$ $=d_{2 n+1}$; this is always possible since $g_{2 n}$ is a polynomial. Let the function $h_{2 n}=\left(z-c_{1}\right)\left(z-c_{2}\right) \cdot \cdots \cdot\left(z-c_{n}\right)$, and consider the functions $g_{2 n}+k_{2 n} h_{2 n}$, for

Received by the editors January 25, 1966. 


$$
\left|k_{2 n}\right|<\frac{1}{(2 n) ! u_{1} u_{2} \cdot \cdots \cdot u_{2 n}}=m_{2 n}
$$

where $u_{i}=\max \left(1,\left|c_{i}\right|\right), 1 \leqq i \leqq 2 n$. These functions map all elements of some neighborhood of $x_{2 n+1}$ into $d_{2 n+1}$, and hence there exists a particular value of $k_{2 n}$ for which $g_{2 n+1}\left(c_{2 n+1}\right)=d_{2 n+1}$, where $f_{2 n+1}$ $=k_{2 n} h_{2 n}, g_{2 n+1}=g_{2 n}+f_{2 n+1}$, and $c_{2 n+1}$ is a member of the dense set $A-\left\{c_{1}, \cdots, c_{2 n}\right\}$.

The functions $\left|f_{j}\right|$ are majorized by

$$
\frac{\left(z-c_{1}\right)\left(z-c_{2}\right) \cdots\left(z-c_{j}\right)}{j ! u_{1} u_{2} \cdot \cdots \cdot u_{j}}=\frac{1}{j !}\left(\frac{z-c_{1}}{u_{1}}\right)\left(\frac{z-c_{2}}{u_{2}}\right) \cdots\left(\frac{z-c_{j}}{u_{j}}\right) .
$$

For each $i, 1 \leqq i \leqq j$, if $\left|c_{i}\right| \leqq 1$, then $u_{i}=1$ and $\left|\left(z-c_{i}\right) / u_{i}\right|=\mid z-c_{i}$ $\leqq|z|+1$, while if $\left|c_{i}\right|>1$, then $u_{i}=\left|c_{i}\right|$ and $\left|\left(z-c_{i}\right) / u_{i}\right| \leqq|z /| c_{i}||$ $+\left|c_{i} /\right| c_{i}||=|z /| c_{i}||+1<|z|+1$. Thus the functions $\left|f_{j}\right|$ are also majorized by $(|z|+1)^{j} / j !$, and therefore $f=\sum_{j=1}^{\infty} f_{j}$ is an entire function with $f\left(c_{i}\right)=d_{i}$ for all $i$. By virtue of the "back-and-forth" induction, the maps $c$ and $d$ are enumerations, i.e., $A=\left\{c_{1}, c_{2}, \cdots\right\}$ and $B=\left\{d_{1}, d_{2}, \cdots\right\}$, since in fact $\left\{c_{1}, \cdots, c_{2 n}\right\} \supseteq\left\{a_{1}, \cdots, a_{n}\right\}$ and $\left\{d_{1}, \cdots, d_{2 n}\right\} \supseteq\left\{b_{1}, \cdots, b_{n}\right\}$ for each $n$. Therefore $f$ takes $A$ on to $B$.

In particular, this gives a negative answer to the question posed by F. Gross in [2]. A more general question remains open, which in one sense is a closer generalization of the first question asked by Erdös:

Let $A$ and $B$ be two denumerable, dense subsets of the complex plane. Does there exist an entire function which maps $A$ onto $B$ and $\bar{A}$ onto $\bar{B}$ ?

According to the proof given above, we can say only that there exists a function whose restriction to $A$ gives a one-to-one map from $A$ to $B$. The author is grateful to the referee for his helpful comments on this paper.

\section{REFERENCES}

1. Paul Erdös, Some unsolved problems, Michigan Math. J. 4 (1957), 291-300.

2. Fred Gross, Function theory, Research Problem 19, Bull. Amer. Math. Soc. 71 (1965), 853.

UNiversity of CALIFornia, Berkeley 